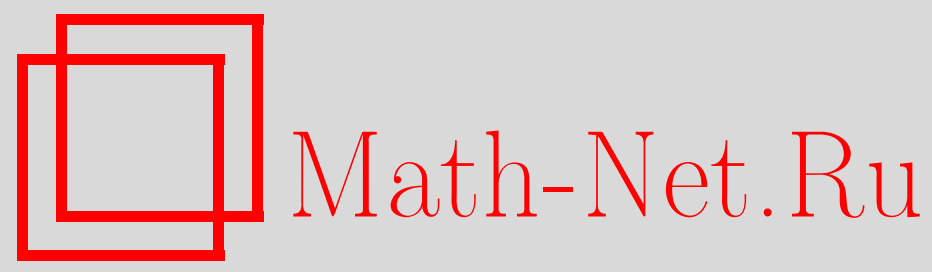

А. А. Коньков, О растущих решениях нелинейных обыкновенных дифференциальных уравнений, Матем. заметки, 1997, том 62, выпуск 5, 792-795

DOI: https://doi.org/10.4213/mzm1666

Использование Общероссийского математического портала Math-Net.Ru подразумевает, что вы прочитали и согласны с пользовательским соглашением http://www . mathnet.ru/rus/agreement

Параметры загрузки:

IP: 52.205 .19 .152

26 апреля 2023 г., 15:26:56

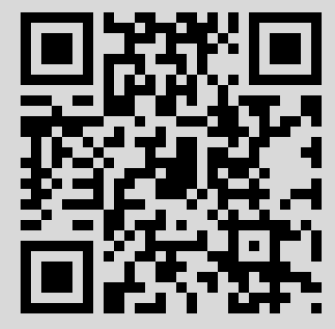




\section{О РАСТУЩИХ РЕШЕНИЯХ НЕЛИНЕЙНЫХ ОБЫКНОВЕННЫХ ДИФФЕРЕНЦИАЛЬНЫХ УРАВНЕНИЙ}

\section{А. А. Коньков}

Рассматриваются решения уравнений порядка $m \geqslant 2$ вида

$$
w^{(m)}=Q\left(r, w, \ldots, w^{(m-1)}\right),
$$

удовлетворяющие условию

$$
w^{(m-i)}\left(r_{*}\right)>\frac{t_{*}}{(i-1) !} r_{*}^{i-1}, \quad i=1, \ldots, m,
$$

где $r_{*} \in(0, \infty), t_{*} \in(0, \infty)$ - некоторые вещественные числа, $Q$ - функция из класса Каратеодори $K_{\text {loc }}\left(\left[r_{*}, \infty\right) \times \mathbb{R}^{m}\right)[1$, c. 9$]$ такая, что для некоторого $k \in\{0, \ldots, m-2\}$ на множестве

$$
\left\{\left(r, t_{0}, \ldots, t_{m-1}\right): r \geqslant r_{*}, t_{m-i} \geqslant \frac{t_{*}}{(i-1) !} r^{i-1}, i=1, \ldots, m\right\}
$$

выполнено неравенство

$$
Q\left(r, t_{0}, \ldots, t_{m-1}\right) \geqslant p(r) g\left(t_{k}\right),
$$

где $p \in L_{\mathrm{loc}}\left(\left[r_{*}, \infty\right)\right), g:(0, \infty) \rightarrow(0, \infty)$ - измеримая функция, причем $p(r) \geqslant 0$ при $r \geqslant r_{*}$ и $g(t) \geqslant g(\tau)$ при $t \geqslant \tau>0$.

Всюду по умолчанию будем считать, что $\eta:[0, \infty) \rightarrow[0, \infty)-$ не равная тождественно нулю монотонно не убывающая функция.

Для всякого вещественного числа $\alpha$ определим функцию $N_{\alpha, \infty}$ равенством

$$
N_{\alpha, \infty}(s)=\int_{1}^{s} \eta(t) g^{-\alpha}(t) t^{\alpha-1} d t
$$

Пусть

$$
\int_{1}^{\infty} \eta(t) g^{-\alpha}(t) t^{\alpha-1} d t<\infty
$$

Положим

$$
N_{\alpha, 0}(s)=\int_{s}^{\infty} \eta(t) g^{-\alpha}(t) t^{\alpha-1} d t
$$

Под $N_{\alpha, \infty}^{-1}$ и $N_{\alpha, 0}^{-1}$ будем соответственно подразумевать функции, обратные к $N_{\alpha, \infty}$ и $N_{\alpha, 0}$.

Наконец, для всякого вещественного числа $\sigma>1$ обозначим

$$
p_{\sigma}(r)=\underset{r / \sigma \leqslant \xi \leqslant \sigma r}{\operatorname{essinf}} p(\xi) .
$$

Эта заметка является логическим продолжением работ [2] и [3], в которых также рассматривались уравнения вида (1), но при несколько иных предположениях относительно $Q$. Ниже изучается следующая проблема: при каких условиях на функции $p$ и $g$ всякое непродолжаемое решение $(1),(2)$ является сингулярным второго рода [1, с. 262$]$. В случае, когда у (1), (2) существуют правильные решения, приводятся оценки для $k$-й производной этих решений.

Работа выполнена при финансовой поддержке Российского фонда фундаментальных исследований, грант № 96-0100653. 
ТЕОРемА 1. Пусть $1<\sigma$ и имеют место соотношения

$$
\begin{gathered}
\int_{1}^{\infty} \eta(t) g^{-1 /(m-k)}(t) t^{1 /(m-k)-1} d t<\infty \\
\int_{r_{*}}^{\infty} \eta\left(\frac{t_{*}}{(m-k-1) !} \xi^{m-k-1}\right) p_{\sigma}^{1 /(m-k)}(\xi) d \xi=\infty
\end{gathered}
$$

Тогда всякое непродолжаемое решение уравнения (1), удовлетворяющее условию (2), является неколеблющимся сингулярным решением второго рода.

TEOpema 2. Пусть $1<\sigma, b \in\left(0, t_{*} /(m-k-1)\right.$ !) $u$

$$
\int_{r_{*}}^{\infty} \eta\left(b \xi^{m-k-1}\right) p_{\sigma}^{1 /(m-k)}(\xi) d \xi=\infty
$$

Тогда найдутся постоянные $A>0, B>0$, зависящ, те только от $\sigma, m, t_{*} / b$, такие, что для всякого правильного решения уравнения (1), удовлетворяющего условию (2), при всех $r$ из некоторой окрестности $\infty$ справедлива оценка

$$
w^{(k)}(r) \geqslant A N_{1 /(m-k), \infty}^{-1}\left(B \int_{r_{*}}^{r} \eta\left(b \xi^{m-k-1}\right) p_{\sigma}^{1 /(m-k)}(\xi) d \xi\right) .
$$

TeOpema 3. Пусть $\alpha \in(0,1 /(m-k)), b \in\left(0, t_{*} /(m-k-1) !\right) u$

$$
\int_{r_{*}}^{\infty} \xi^{\alpha(m-k)-1} \eta\left(b \xi^{m-k-1}\right) p^{\alpha}(\xi) d \xi=\infty .
$$

Тогда найдутся постоянные $A>0, B>0$, зависящие только от $\alpha, m, t_{*} / b$, такие, что для всякого правильного решения уравнения (1), удовлетворяющего условию (2), при всех $r$ из некоторой окрестности $\infty$ справедлива оценка

$$
w^{(k)}(r) \geqslant A N_{\alpha, \infty}^{-1}\left(B \int_{r_{*}}^{r} \xi^{\alpha(m-k)-1} \eta\left(b \xi^{m-k-1}\right) p^{\alpha}(\xi) d \xi\right) .
$$

ЗАмЕЧАнИЕ 1. Можно показать [4], что если у уравнения (1) существует правильное решение, удовлетворяющее условию (2), и при этом для некоторого $\alpha \in(0,1 /(m-k))$

$$
\int_{r_{*}}^{\infty} \xi^{\alpha(m-k)-1} \eta\left(\frac{t_{*}}{(m-k-1) !} \xi^{m-k-1}\right) p^{\alpha}(\xi) d \xi=\infty
$$

то

$$
\int_{1}^{\infty} \eta(t) g^{-\alpha}(t) t^{\alpha-1} d t=\infty
$$

Поэтому сушествует пара окрестностей бесконечно удаленной точки, между которыми отображение $N_{\alpha, \infty}$ устанавливает взаимно однозначное соответствие. Тем самым, правая часть (7) корректно определена. 
Tеорема 4. Пусть $1<\sigma, b \in\left(0, t_{*} /(m-k-1) !\right)$, uмеeт место (4) $u$

$$
\int_{r_{*}}^{\infty} \eta\left(b \xi^{m-k-1}\right) p_{\sigma}^{1 /(m-k)}(\xi) d \xi<\infty
$$

Тогда найдутся постоянные $A>0, B>0$, зависящ, те только от $\sigma, m, t_{*} / b$, такие, что для всякого правильного решения уравнения (1), удовлетворяющего условию (2), при всех $r$ из некоторой окрестности $\infty$ справедлива оченка

$$
w^{(k)}(r) \leqslant A N_{1 /(m-k), 0}^{-1}\left(B \int_{r}^{\infty} \eta\left(b \xi^{m-k-1}\right) p_{\sigma}^{1 /(m-k)}(\xi) d \xi\right) .
$$

Tеорема 5. Пусть $\alpha \in(0,1 /(m-k)), b \in\left(0, t_{*} /(m-k-1) !\right)$, uмеeт место (3) $u$

$$
\int_{r_{*}}^{\infty} \xi^{\alpha(m-k)-1} \eta\left(b \xi^{m-k-1}\right) p^{\alpha}(\xi) d \xi<\infty .
$$

Тогда найдутся постоянные $A>0, B>0$, зависящие только от $\alpha, m, t_{*} / b$, такие, что для всякого правильного решения уравнения (1), удовлетворяющего условию (2), при всех $r$ из некоторой окрестности $\infty$ справедлива оченка

$$
w^{(k)}(r) \leqslant A N_{\alpha, 0}^{-1}\left(B \int_{r}^{\infty} \xi^{\alpha(m-k)-1} \eta\left(b \xi^{m-k-1}\right) p^{\alpha}(\xi) d \xi\right) .
$$

ТЕОрема 6. Предположим, что $p$ не возрастает, $b \in\left(0, t_{*} /(m-k-1) !\right) u$

$$
\int_{r_{*}}^{\infty} \eta\left(b \xi^{m-k-1}\right) p^{1 /(m-k)}(\xi) d \xi=\infty
$$

Тогда найдутся постоянные $A>0, B>0$, зависящие только от $m, t_{*} / b$, такие, что для всякого правильного решения уравнения (1), удовлетворяющего условию (2), при всех $r$ из некоторой окрестности $\infty$ справедлива оценка

$$
w^{(k)}(r) \geqslant A N_{1 /(m-k), \infty}^{-1}\left(B \int_{r_{*}}^{r} \eta\left(b \xi^{m-k-1}\right) p^{1 /(m-k)}(\xi) d \xi\right) .
$$

Теорема 7. Предположим, что р не возрастает, $b \in\left(0, t_{*} /(m-k-1) !\right)$, имеет место (4) $u$

$$
\int_{r_{*}}^{\infty} \eta\left(b \xi^{m-k-1}\right) p^{1 /(m-k)}(\xi) d \xi<\infty .
$$

Тогда найдутся постоянные $A>0, B>0$, зависящие только от $m, t_{*} / b$, такие, что для всякого правильного решения уравнения (1), удовлетворяющего условию (2), при всех г из некоторой окрестности $\infty$ справедлива оченка

$$
w^{(k)}(r) \leqslant A N_{1 /(m-k), 0}^{-1}\left(B \int_{r}^{\infty} \eta\left(b \xi^{m-k-1}\right) p^{1 /(m-k)}(\xi) d \xi\right) .
$$


ЗАмЕчАниЕ 2 . Пусть для любого $\tau_{*}>0$ найдется функция $Q^{*} \in L_{\mathrm{loc}}\left(\left[r_{*}, \infty\right)\right)$ такая, что $Q\left(r, t_{0}, \ldots, t_{m-1}\right) \geqslant Q^{*}(r) \geqslant 0$ на множестве

$$
\left\{\left(r, t_{0}, \ldots, t_{m-1}\right): r \geqslant r_{*}, t_{m-i} \geqslant \frac{\tau_{*}}{(i-1) !} r^{i-1}, i=1, \ldots, m\right\}
$$

и

$$
\int_{r_{*}}^{\infty} Q^{*}(r) d r=\infty
$$

Тогда в теоремах 1-7 условие (2) может быть заменено на условие

$$
w^{(m-i)}\left(r_{*}\right)>0, \quad i=1, \ldots, m \text {. }
$$

Также в теореме 1 соотношение (5) может быть заменено на соотношение (6), в котором $b \in(0, \infty)$.

Более того, в теоремах 2-7 ограничение вида $b \in\left(0, t_{*} /(m-k-1) !\right)$ на вещественное число $b$ может быть ослаблено до ограничения $b \in(0, \infty)$. При этом постоянные $A>0$, $B>0$ в теоремах 2 и 4 будут зависеть только от $\sigma, m$, в теоремах 3 и 5 - только от $\alpha, m$, а в теоремах 6 и 7 - только от $m$.

ПРИмеР. Рассмотрим уравнение

$$
w^{(m)}=a_{0} e^{(1-\lambda) r} w^{\lambda}
$$

где $a_{0}>0, \lambda>1$ - некоторые вешественные числа. Полагая в теореме $7 k=0, g(t)=t^{\lambda}$, $p(\xi)=e^{(1-\lambda) \xi}, \eta(\xi)=1$, для всякого решения уравнения (10), удовлетворяющего условию $(9)$, будем иметь при всех $r$ из некоторой окрестности $\infty$

$$
w(r) \leqslant C e^{r}
$$

где константа $C>0$ зависит только от $m, \lambda$. Несложно убедиться, что функция $w(r)=a e^{r}$ при подходящем выборе константы $a>0$ будет решением (10), (9).

Таким образом, в данном примере оценка (8) оказывается более точной, чем соответствуюшие оценки, содержашиеся в [1]-[3], [5].

\section{СПИСОК ЦИТИРОВАННОЙ ЛИТЕРАТУРЫ}

1. Кигурадзе И.Т., Чантурия Т. А. Асимптотические свойства решений неавтономньх обыкновенных дифференциальных уравнений. М.: Наука, 1990. 2. Коньков А. А. // Матем. заметки. 1996. Т. 60. № 4. С. 616-620. 3. Коньков А. А. О поведении решений нелинейных обыкновенных дифференциальных уравнений // Докл. РАН. (в печати). 4. Коньков А. А. // Дифференц. уравнения. 1996. Т. 32. №6. С. 854. 5. Рабцевич В. А. // Дифференц. уравнения. 1994. T. 30. № 3. C. $400-404$. 